\title{
11 Closing the gap in programming for adolescents living with HIV in Eastern and Southern Africa
}

\author{
The role of social protection in \\ positive prevention
}

\author{
Nompumelelo Zungu, Elona Toska, Lesley Gittings \\ and Rebecca Hodes
}

\section{Introduction}

The Eastern and Southern Africa (ESA) region has a high burden of HIV and AIDS, with an estimated 19.6 million people living with the virus (UNAIDS, 2018). All countries in the region have 'generalised' epidemics, with an adult prevalence of $6.8 \%$ among those aged 15-49 (greater variations are observed between countries). HIV incidence and prevalence tends to be concentrated in particular sub-populations such as adolescent girls and young women (AGYW), men who have sex with men (MSM), transgender people, people who sell sex, prisoners and people who inject drugs (UNAIDS, 2018). According to UNICEF, 2.1 million adolescents aged 10-19 were living with HIV globally in 2016. Furthermore, 590,000 young people between the ages of 15 and 24 were newly infected with HIV, of whom 250,000 were adolescents between the ages of 15 and 19 in 2017 (UNAIDS, 2018). AGYW aged 15-24 are the most affected by HIV: HIV prevalence among this population in the ESA region was double that among adolescent boys and young men (ABYM) in 2016 (3.4\% vs 1.6\%) (UNAIDS, 2017; Govender et al., 2018). Furthermore, while HIV incidence rates are generally decreasing, they are still high in many ESA countries (UNAIDS, 2017). It is estimated that new HIV infections declined by $56 \%$ among children (aged 14 or younger) to 77,000 between 2010 and 2016 (UNAIDS, 2017). Among adults, declines were estimated at $29 \%$ over the same period, although significant variations are observed between countries.

In light of the foregoing, UNICEF (2017) has estimated that, without concerted action, the actual number of new adolescent infections will increase steadily in the next decade. In addition, in the last decade, reductions in AIDS-related sickness and death among children and adolescents in the region have demonstrated the vital impact of national ART programmes. While remarkable progress has been made in preventing paediatric HIV through the provision of prevention of mother-to-child transmission treatment, HIV testing and ART initiation and adherence, support for children 
and adolescents is lacking. Figures from UNICEF (2017) suggest that only $43 \%$ of infants exposed to HIV are tested within two months, and that only $43 \%$ of the 2.1 million children living with HIV globally are receiving ART. Thus, while HIV testing and treatment for infants has improved in recent years, the benefits of these programmes tapers off for older children and adolescents.

Limited available data for adolescents suggest worrisome trends regarding treatment access and AIDS-related deaths (UNAIDS, 2017). According to UNAIDS (2018), only 67 countries worldwide reported disaggregated adolescent treatment data. Among these, median ART coverage was low, at $20 \%$ in 2016. These data suggest that adolescents in ESA are the only population group in the region among which AIDS-related morbidity and mortality continues to grow (UNAIDS, 2018). The total numbers of adolescents living with HIV are growing, with a greater proportion of those infected through mother-to-child transmission surviving into adulthood due to improved access to ART, while new infections among 15-24-year-olds persist (UNAIDS, 2017).

The findings of the HPTN052 study (showing that ART can prevent onward transmission among HIV-discordant individuals) have challenged the assumption that HIV treatment and prevention are distinct, with a growing body of research demonstrating how effective HIV treatment and prevention are intrinsically connected (Cohen et al., 2013). This evidence, coupled with the UNAIDS 90-90-90 targets and the recent move to adopt a 'test and treat' approach in many countries with high HIV prevalence and incidence, has led to the promotion of ART as means of enabling 'positive prevention' among key populations and vulnerable groups such as AGYW aged 15-24. The current, burgeoning evidence base on associations between social protection and improvements in health outcomes among HIV-positive children and adolescents opens up a new field of prospective enquiry and promising prospective interventions.

There is growing evidence that social protection programmes have the potential to reduce HIV transmission through promoting safer sexual behaviours among adolescents and promoting adherence to ART. UNICEF defines social protection as 'a set of public and private policies and programmes aimed at preventing, reducing and eliminating economic and social vulnerabilities to poverty and deprivation' (UNICEF, 2015). It includes both formal and informal initiatives that provide social assistance to extremely poor individuals and households, with an aim of mitigating risks and related consequences (Devereux and Sabates-Wheeler, 2004). Social protection can be provided through public (governmental and non-governmental) and private institutions and may fulfil protective, promotive, preventative or transformative capacities. In this chapter, social protection is conceptualised broadly to include many possible interventions and components that fall under this umbrella term. Specifically, this chapter focuses on the burgeoning evidence base on associations between access 
to social grants and other forms of social protection and the improved positive health outcomes among ALHIV. A transdisciplinary research base that captures the impact of social protection initiatives on health behaviours demonstrates the intertwining of health and social domains in global development, and the responsiveness of each to boundary-spanning interventions that may target either health or social development, but whose benefits ripple widely from their central location to other dimensions of life, well-being and livelihood.

We employ a twofold conceptualisation of HIV prevention, treatment and care package for adolescents living with HIV (Gittings et al., 2016), as both the prevention of new HIV-infections among those previously HIVnegative and as the prevention of onward transmission of HIV - "positive prevention'. This chapter makes the case for elaborating the positive protection approach, drawing on existing HIV-oriented social protection programmes. The chapter draws on previous work about the potential of social protection to support positive prevention and adherence to ART for children and adolescents living with HIV (Gittings et al., 2016; Toska et al., 2017a). In doing so, it highlights the urgent need for flexible, inclusive and responsive social protection mechanisms as a promising and productive means of promoting improved health outcomes and reducing risk behaviours among ALHIV.

\section{ART adherence, sexual practices and reproductive health among ALHIV in ESA}

A growing evidence base shows that biomedical interventions combined with social programmes are the best means of reducing new HIV infections and, ultimately, turning the tide of the AIDS epidemic (Padian et al., 2010), especially among young people (Cluver et al., 2016a). However, while reducing incidence through keeping adolescents HIV-free is critical, it is also important to support those already living with HIV to lead safe and healthy lives, including through viral suppression, engaging in safer sex and having planned, safe pregnancies and parenthoods. This section briefly reviews factors related to non-adherence and sexual risk-taking among adolescents living with HIV. It describes how different forms of social protection may help to support ART-adherence and reduce sexual risk-taking among adolescents in hyper-endemic contexts.

ART offers an opportunity for the survival and long-term well-being of people living with HIV. Yet many of the estimated 1.2 million children and adolescents living with HIV in ESA struggle to initiate and remain on ART. Once initiated on ART, $80 \%-95 \%$ adherence is required to avoid medication resistance, meaning that even occasional lapses in adherence to the ART regimen could reduce its efficacy (Paterson et al., 2000). Promisingly, increased linkages to ensure that adolescents access both sexual and reproductive health (SRH) and HIV services have been shown to increase 
HIV-positive adolescents' access to and use of treatment services (UNICEF, 2015) and healthcare (Hodes et al., 2018a, 2018b).

Understanding the sexual practices and reproductive health needs of adolescents living with HIV remains critical for positive prevention. A recent systematic review focusing on sexual risk-taking among adolescents found mostly cross-sectional evidence on eight sexual risk-taking outcomes in 13 ESA countries (Toska et al., 2017b). Studies reported widely varying rates of sexual risk-taking, with nearly half of adolescents being sexually active, $33 \%-50 \%$ reporting unprotected sex, and 10\%-66\% reporting other sexual risk practices such as transactional sex, multiple sexual partners in the last year or sex with older men (Toska et al., 2017b). Adolescent girls living with HIV reported higher prevalence of transactional sex, unprotected sex and sex with older partners, though adolescent boys were more likely to report early sexual debut and multiple sexual partners.

\section{Social, economic and structural drivers of sexual risk behaviours and non-adherence to ART}

The limited research on adolescent ART adherence shows that many adolescents struggle to comply with ART regimens (Auld et al., 2014) and reveals exceptionally low rates of children and adolescents maintaining ART adherence (Cluver et al., 2016b; Hudelson \& Cluver, 2015; Nachega et al., 2009). Evidence suggests that a combination of factors, including lack of social support, disclosure challenges, food insecurity and the costs of clinic attendance make it difficult for adolescents to initiate and remain on treatment (Cluver et al., 2016b; Hudelson \& Cluver, 2015; Nachega et al., 2009). A burgeoning literature documents the additional, various experiential, clinical and operational challenges that adolescents living with HIV face in engaging with health programmes (Bernays et al., 2016; DelanyMoretlwe et al., 2015; Toska et al., 2015; Vale, Hodes \& Cluver, 2017; Visser, Zungu \& Ndala-Magoro, 2015).

Drivers of ART non-adherence and sexual risk-taking among adolescents living with HIV include (1) social, economic and structural issues; (2) nondisclosure, stigma and discrimination; (3) caregiver well-being, disrupted family structures and caregiver-child relationships; and (4) healthcare services and health systems factors (Campbell et al., 2012; Gittings et al., 2016; Hudelson \& Cluver, 2015). A growing literature also demonstrates the interconnections between structural deprivations, non-adherence to ART and sexual risk-taking (Kidman et al., 2018; Toska \& Cluver, 2018). This evidence is presented below, providing an overview on how structural drivers, stigma and discrimination, caregiver relationships and family structure, and health services are linked to both poor ART adherence and sexual risk outcomes.

Social, economic, and structural drivers - Poverty and related food insecurity are known barriers to ART adherence. Many adolescents living with HIV in ESA face food insecurity, and believe that these medicines must 
be taken with food (Gittings et al., 2016; WHO, 2015). Food insecurity and poverty are also known drivers of HIV risk behaviours for adolescent girls because they promote high-risk relationships with older partners, in which transactional sex provides the means of subsistence or survival for girls and young women. These vulnerabilities are mutually reinforcing, fostering a concatenation of risky behaviours, including unprotected sex and ART non-adherence. Understanding the gendered dimension of ART nonadherence for adolescents is also important because certain social protection interventions may affect boys and girls differently (Cluver et al., 2016b; Handa et al., 2014), and a higher proportion of new adolescent infections occur in girls (UNAIDS, 2017). Given that age-disparate sex is a driver of these infections, work with their older male partners on safer sexual practices and improved ART adherence is also warranted.

A recent study found that exposure to violence - at home and school and in clinics - was strongly linked with increased ART non-adherence, as shown in Figure 11.1 (Cluver et al., 2018), with exposure to multiple types of violence associated with higher levels of ART non-adherence. The study focuses on the detrimental effects of clinic victimisation in particular, identifying the negative impact that punitive, debasing and violent healthcare services have on both healthcare attendance and compliance with medical regimens among adolescents. Regarding other forms of violent victimisation beyond healthcare facilities, several studies in Uganda and Kenya reported that gender-based violence was linked with unintended pregnancies (Nhamo, 2013; Obare, Birungi \& Kavuma, 2011) and transactional sex (Nhamo, 2013). The association between violence and ART non-adherence requires further investigation.

Disclosure, stigma and discrimination - Social barriers to treatment must be challenged, particularly HIV-related stigma and discrimination and legislation addressing the criminalisation of HIV non-disclosure, onward transmission, and age of consent to access testing services and criminalising same-sex practices through legislation. Evidence from communitybased HIV testing programmes suggests that removing such barriers would encourage more people to get tested and seek out treatment (Iwuji et al., 2018; Orne-Gliemann et al., 2015). For fear of unwanted disclosure, some adolescents and young adults may not adhere to medicines when they are in situations where they might be seen taking medicines (leading to unintended HIV-positive serostatus disclosure) - such as in public, or even in social situations and with friends, family members or intimate partners (Hodes et al., 2018a). This issue may be compounded by fear of loss of material support in situations in which adolescents are also receiving material support from their partners (Toska et al., 2015) and family or community members.

Negative effects on adherence have also been seen in children and adolescents who have not been disclosed to at an appropriate age, defined by WHO as starting at the age of 10 and completed by the age of 12 (Cluver et al., 2015; Hudelson \& Cluver, 2015). This may be due to poor understanding of 


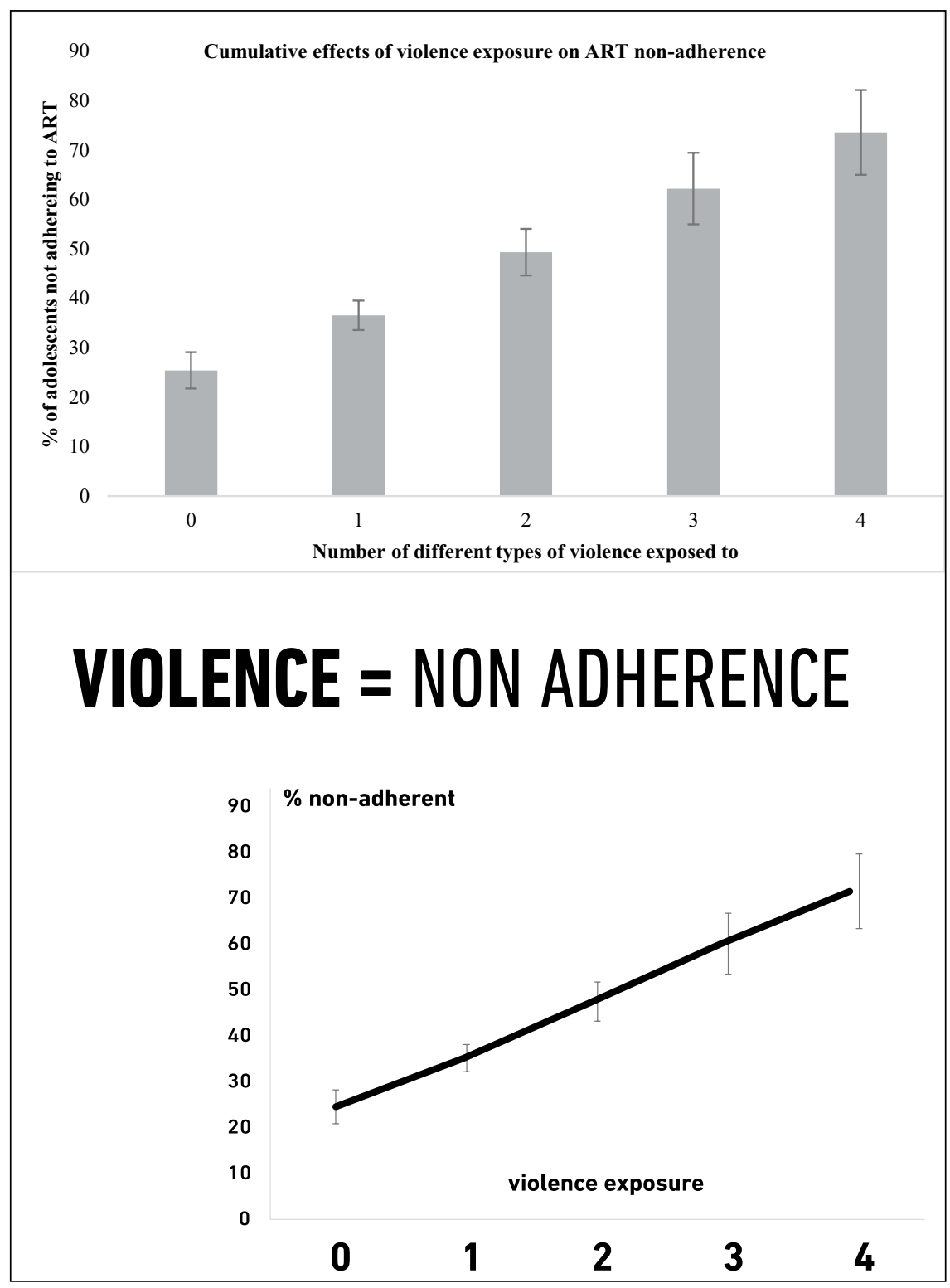

Figure 11.1 Marginal effects model testing for additive effect of violence on adolescent ART adherence (Cluver et al., 2018 - figure INCLUDED with AUTHOR permission).

Source: Cluver et al. (2018). 
the reasons for which they are taking medication or issues with acceptance of their HIV-positive status. By contrast, adolescents who were disclosed to early, and in a sensitive and supportive way, achieve better adherence and sexual health outcomes (Cluver et al., 2015; Toska et al., 2015).

Caregiver relationships and family structures - Living alone or with a partner (compared to living with a caregiver) were strongly associated with sexual risk-taking among adolescents living with HIV in two studies in Uganda (Baryamutuma et al., 2010; Mbalinda et al., 2015). Moreover, caregiver monitoring is closely linked to safe sexual practices among adolescents living with HIV (Toska et al., 2017a). For this reason, it has been suggested that caregiver psychosocial and physical well-being is fundamental to supporting ART adherence and safe sexual practices (Cluver et al., 2018; Hudelson \& Cluver, 2015; Toska et al., 2017a; WHO, 2015).

Healthcare services and system factors - There are also many documented healthcare-related barriers to ART adherence, including treatment costs, travel costs and distances to clinics and transition between paediatric and adult care (Cluver et al., 2018; Hudelson \& Cluver, 2015; PATA \& WHO, 2015). Additionally, accessing HIV and reproductive health services separately can be stigmatising, which is a core reason for integration of HIV and sexual healthcare to support all SRH services to be HIV-inclusive rather than HIV-specific ${ }^{1}$ (Hodes, 2013; Gittings et al., 2016). HIV-related stigma and discrimination can exacerbate social exclusion and create barriers to the uptake of health services, particularly among adolescents. Transformative social protection aims to create enabling legal and normative environments for the delivery of provisions that address stigma and reduce barriers to uptake of health services (Gittings et al., 2016).

Syndemic risk factors for ART adherence and sexual risk-taking - The research among ALHIV in ESA outlined in the section below suggests a strong overlap between factors shaping ART adherence, sexual risk-taking and retention in HIV/SRH care in this vulnerable group of young people. Many of these factors are shared for both of these health outcomes and are closely linked to structural vulnerabilities. Given that non-adherence combined with risky sexual practices fosters high-risk situations for HIV onward transmission, efforts to improve adherence must go hand in hand with those aimed at promoting safer sex.

\section{Social protection to interrupt pathways for non-adherence and sexual risk-taking - a review of the evidence}

A growing body of literature demonstrates that social protection is a critical enabler for HIV prevention outcomes through interrupting pathways for non-adherence and sexual risk-taking (Baird et al., 2012; Gittings et al., 2016). There is also a strong evidence base that shows the impacts of social protection on HIV and risky-sex pathways such as food insecurity, early marriage, economic migration, transactional sex, early pregnancy, early 
sexual debut and school dropout (Gittings et al., 2016; Shisana et al., 2014; UNICEF, 2015). This section provides an overview of this evidence.

In 2016, a review was conducted on the role of social protection for improved adherence and HIV-related health outcomes among children and adolescents in ESA that extended and detailed these findings. The review found 11 programmes, including one RCT (Ssewamala et al., 2018), one pre-post pilot (Bhana et al., 2014), one pre-post small-scale pilot RCT (Snyder et al., 2014), two quantitative (Grimwood et al., 2012; Van Winghem et al., 2008) and six qualitative studies (Busza et al., 2014; Denison et al., 2015; Mupambireyi et al., 2014; O’Hare et al., 2005; Strasser \& Gibbons, 2014) for which there was evidence of ART adherence outcomes among children aged 15 or younger) and/or adolescents (aged 10-19). The majority of these included adherence alongside other outcomes, such as HIV treatment knowledge, psychosocial well-being and retention in care (Gittings et al., 2016).

A more recent systematic review of ART adherence interventions among adolescents living with HIV found only one additional new programme to support ART adherence among adolescents living with HIV, although a study conducted in Thailand provided useful insights into the potential of group-based adherence counselling (Ridgeway et al., 2018).

A 2017 systematic review of programmes to prevent or respond to sexual risk-taking among adolescents living with HIV located four interventions. Three of the included intervention studies were individual-level randomised controlled trials (Lightfoot, Rotheram-Borus \& Tevendale, 2007; Nhamo, 2013; Senyonyi et al., 2012), with one pre- and post-test experimental design study (Snyder et al., 2014). All four studies reported increases in condom use following the intervention; however, these were only significant in one intervention (Nhamo, 2013). The social protection interventions included in these studies were group-based support, livelihoods training, and integrated HIV/SRH services.

\section{What types of social protection work?}

Among different types of social protection, cash transfers have received significant attention, and there is a rich multidisciplinary literature on their efficacy. 'Cash transfers', in the form of social support grants, travel vouchers to get to facilities and school lunch programmes, can support adherence by addressing poverty-related factors, such as food insecurity and household unemployment (Cluver et al., 2018). In addition, evidence demonstrates that social transfers in the form of transport costs and food can have an effect on adult ART adherence (UNAIDS, 2014). However, the current literature is unclear on whether 'cash-only' social protection interventions directly impact adolescent adherence to ART. The only intervention that focused on ART adherence is a cash plus care plus capability intervention trial in Uganda, which focuses directly on adherence and viral suppression 
(Bermudez et al., 2018; Ssewamala et al., 2018). Two additional pre- and post-test small-scale trials including adherence outcomes had promising results (Bhana et al., 2013; Snyder et al., 2014). Cross-sectional analyses from a large cohort in South Africa found that improving food security was associated with reduced ART non-adherence (Cluver et al., 2016b). Within this study, having sufficient funding to get to the clinic was also associated with improved retention on treatment (Cluver et al., 2018).

Among trials and evaluations of social cash transfers, seven studies found improvements in HIV-related treatment knowledge and adherence (Gittings et al., 2016). Twelve studies included children and adolescents living with HIV, and two of these were quantitative studies of care only that focused on improving ART-related outcomes (Grimwood et al., 2012; Van Winghem et al., 2008). The other two were pre-post pilot studies of care-only interventions (Bhana et al., 2013; Snyder et al., 2014). One trial among adolescent girls living with HIV in Zimbabwe combined cash with care interventions (Nhamo, 2013), resulting in improved condom use and reduced transactional sex. Additional analyses from a large cohort in South Africa suggests that attending fee-free schools combined with care interventions at home and clinics was strongly associated with reduced unprotected sex, especially among adolescent girls living with HIV (Toska et al., 2017a). These last two studies demonstrate associations between social protection interventions and the enhanced capacity for 'positive prevention' among ALHIV.

Beyond cash social protection, care mechanisms offer value in several ways: through directly benefitting recipients, acting as flexible mechanisms that can respond to their shifting, complex needs, in supporting the uptake and retention of other types of social protection (Gittings et al., 2016). Care provisions improved adherence in two pre- and post-test small-scale pilot trials (Bhana et al., 2013; Snyder et al., 2014), three quantitative studies (Cluver et al., 2016a; Grimwood et al., 2012; Van Winghem et al., 2008) and six qualitative studies (Busza et al., 2014; Denison et al., 2015; Mupambireyi et al., 2014; Parker et al., 2013; Strasser and Gibbons, 2014).

The potential of home-based and community care interventions has been demonstrated. One study found an association between receiving a community care intervention and reduced probabilities of attrition and mortality (Grimwood et al., 2012). A study by Busza and colleagues found that home-based care support was positively received by families of children and younger adolescents living with HIV in Tanzania (Busza et al., 2014). HIV-specific support groups have shown increased linkages to healthcare for adolescents living with HIV (Snyder et al., 2014), and peer-education interventions have also demonstrated improved adherence support (Denison et al., 2015). Such home- and community-based care programmes may improve ART adherence by providing psychosocial support, replacing costs of traditional healthcare (facility-based care) and enabling beneficiaries access to livelihood opportunities and life-saving health services (Busza et al., 2014; Gittings et al., 2016; Grimwood et al., 2012). There is also a 
much larger literature on community and home-based care initiatives for HIV testing and treatment (Hodes and Naimak, 2011; Schneider, Hlophe and van Rensburg, 2008) preceded in South Africa by community-based healthcare interventions reaching back over half a century (Phillips, 2014).

Three group and individual counselling-based programmes to reduce sexual risk-taking among adolescents living with HIV in Kenya and Uganda showed promising reductions in unprotected sex and multiple sexual partners. Last, capability interventions (i.e. programmes that support skills development and advance livelihoods) demonstrate promise (Gittings et al., 2016). For some adolescents, accessing interventions that support their capabilities may support ART adherence through better financial outcomes and therefore improve food and clinic access and reduce riskier sex.

Evidence also demonstrates that social protection, particularly cash plus care provisions in combination, have greater potential for improved HIV-related health outcomes among adolescents than cash interventions alone (Bandiera et al., 2012; Cho et al., 2011; Cluver et al., 2016a; Duflo, Dupas \& Kremer, 2011; Karim, 2015). It has been suggested that social protection interventions with multiple components have additive and possibly multiplicative effects (Cluver et al., 2016a) and are necessary to meet the complex psychosocial needs of adolescents living with HIV (Amzel et al., 2013). Combinations can respond to the manifold pathways to ART nonadherence, in acknowledgement of the myriad intersecting challenges that adolescents may face to adherence. Promisingly, there is evidence that improving food security alone, and combined with other forms of social protection, significantly affected adherence outcomes for adolescents living with HIV (Cluver et al., 2016b). Similarly, combining care interventions (good caregiver monitoring) with clinic care (adolescent-sensitive services) and cash interventions (access to fee-free schools) reduced unprotected sex reported by adolescent girls living with HIV (Gittings et al., 2016).

\section{How affordable and feasible is social protection?}

The relative affordability, acceptability and scalability of social protection provisions are common concerns for ESA governments and for bilateral agencies. Social protection initiatives for children and adolescents are an investment in their health and well-being. They are also a long-term cost-saving mechanism through off- setting the costs of poor health outcomes and capitalising further on earlier investments in public health (Remme etal., 2014). The World Bank Social Safety Nets report (2015) examined social assistance in 120 developing countries and suggested that well-designed social assistance programmes are cost-effective (between $1.5 \%$ and $1.9 \%$ of GDP). Social assistance in sub-Saharan Africa only covers one-tenth of the poorest 20\% (Honorati, Gentilini \& Yemtsov, 2015), and expanding social protection provisions is possible for most African countries (Garcia \& Moore, 2012). Co-financing from multiple government departments 
(as demonstrated in the STRIVE consortium) can make budgetary commitments for social protection more manageable but requires integrating evaluations and budgeting mechanisms. It also requires a conceptual shift in the cost-benefit analysis of health and social programming, similar to what was required in the reconceptualisation of the global ART rollout as not just a social and medical good but an investment in public health which would benefit the fiscus over the long term. Recent cost-benefit modeling from South Africa suggests that an investment of ZAR 4154.22 (USD 299.73) per adolescent living with HIV per year would allow for $69.5 \%$ to be retained in care (Standish-White, 2018).

\section{Conclusion and recommendations}

In view of the potential linkages between poor adherence and sexual risktaking among ALHIV (Kidman et al., 2018; Marhefka et al., 2010; Toska \& Cluver, 2018), it is of crucial importance to identify policy and programmatic interventions that can address their vulnerabilities in order to improve their health and resilience and to prevent the onward transmission of HIV. Our analysis of key findings in the literature suggests that flexible and responsive social protection mechanisms can be an important component in the response to the complex causal pathways of HIV risk. Evidence on social protection programmes and policies highlights the potential for combinations of social protection interventions, particularly 'cash' combined with 'care' and 'capability', to interrupt pathways for sexual risk behaviours and non-adherence to ART and to foster resilience.

The focus of this chapter is not only on social protection programmes and policies that help HIV-negative children and adolescents stay healthy, but also that support children and adolescents living with HIV to take medication and prevent transmitting HIV onwards. There is a small body of literature on positive prevention, ART adherence and social protection among adolescents in ESA. However, a review of evidence suggests a number of overlapping mechanisms through which social protection may achieve these impacts: (1) poverty reduction and economic development (Gillespie et al., 2007; Nattrass \& Gonsalves, 2009); (2) improved educational outcomes such as school retention for girls and young women (Pettifor et al., 2008; UNAIDS, 2014; UNICEF, 2015); (3) improved food security (Cluver et al., 2016b; Emenyonu et al., 2010); and (4) improved psychosocial outcomes (Govender et al., 2014). Investigating overlapping mechanisms and additive effects of health and social interventions signals a move away from single-source, siloed and isolated interventions towards a more comprehensive, collective and broadscale conceptualisation of the boundary-spanning, complementary and interlinked effects of development work across the domains of health and social development.

Available evidence indicates the potential of social protection for alleviating structural drivers of HIV risk, including poverty and unemployment, gender inequalities and social stigma and marginalisation. If social 
protection is to be effective for children and adolescents, it must be both HIV-inclusive and responsive to their developmental and contextual needs (Delany-Moretlwe et al., 2015; Pettifor et al., 2015). A key area for further inquiry is improving our understandings of how different combinations of social protection programmes can have the most impact for the prevention of new HIV infections as well as 'positive prevention' among adolescents living with HIV.

Global strategies such as the Fast Track targets and policy directives such as the Sustainable Development Goals offer a breadth of opportunity for scaling up more holistic forms of social protection. These development plans commit governments, donors and bilateral partners to ambitious, large-scale improvements in health and social service provision, and they are often echoed by the state's own policy provisions and commitments, reflected, for instance, in the objectives of South Africa's National Development Plan (National Planning Commission, 2012).

The literature surveyed in this chapter suggests that different forms of social protection can support improved ART adherence and reduce risky sexual practices among adolescents living with HIV. This is promising in achieving the dual objectives of keeping young people living with HIV healthy while reducing the risk of onward transmission. However, the potential pathways through which different types of social protection succeed for these adolescents requires further, careful elucidation. For policy and programming, questions about which forms of social protection offer the greatest benefit (and calculations of their cost-effectiveness) are pressing. Further research is urgently needed to address these questions. As a large cohort of HIV-affected children in sub-Saharan Africa reaches adolescence, social protection that improves the resilience of young people is also needed, particularly a combination of social protection which includes 'capabilities' (or skills building) components in addition to 'cash' and 'care' provisions.

While cash transfers have received much attention, this chapter argues for greater focus on care and capability interventions, either alone or in combination. Here, novel combinations of biomedical and social interventions that factor behavioural alongside structural, psychosocial and biomedical responses are a new direction in HIV research (Coates, Richter \& Caceres, 2008), as seen in the DREAMS initiative (Gittings et al., 2016; PEPFAR, 2015). However, questions of scalability, flexibility and sustainability of such interventions remain (Delany-Moretlwe et al., 2015). Ensuring reach and uptake for the most vulnerable will be integral to ensuring that no child or adolescent is left behind in the future HIV response.

In conclusion, social protection's potential to address structural drivers of vulnerabilities places such provisions in a unique position to address multiple health outcomes for adolescents living with HIV in ESA. Additional empirical and programmatic evidence is needed to explore which combinations are most effective for different sub-groups of adolescents, including for highly vulnerable key populations which face additional structural and socio-legal barriers to accessing services. Such evidence should be 
complemented by rigorous cost-effectiveness analyses to support informed decision-making on which provisions are likely to have the highest return on investment, and an adaptive approach that matches social protection provisions to the profile and needs of each subgroup, shifting from care plus cash to care plus cash plus capability combinations, as children and adolescents living with HIV become older and aspire for a future in which they are not just surviving, but thriving.

\section{Note}

1 HIV-specific social protection is tailored specifically to HIV services, whereas HIVinclusive social protection includes but is not limited specifically to HIV. For this reason, HIV-specific social protection can be stigmatizing.

\section{References}

Amzel, A., Toska, E., Lovich, R., Widyono, M., Patel, T., Foti, C., Dziuban, E.J., Phelps, B. R., Sugandhi, N., Mark, D. and Altschuler, J., 2013. Promoting a combination approach to paediatric HIV psychosocial support. AIDS, 27(Suppl 2), pp. S147-S157.

Auld, A.F., Agolory, S.G., Shiraishi, R.W., Wabwire-Mangen, F., Kwesigabo, G., Mulenga, M., Hachizovu, S., Asadu, E., Tuho, M.Z., Ettiegne-Traore, V. and Mbofana, F., 2014. Antiretroviral therapy enrollment characteristics and outcomes among HIV-infected adolescents and young adults compared with older adults - seven African countries, 2004-2013. Morbidity and Mortality Weekly Report, 63(47), pp. 1097-1103.

Baird, S.J., Garfein, R. S., McIntosh, C. T. and Özler, B., 2012. Effect of a cash transfer programme for schooling on prevalence of HIV and herpes simplex type 2 in Malawi: A cluster randomised trial. Lancet, 379(9823), pp. 1320-1329.

Bandiera, O., Buehren, N., Burgess, R., Goldstein, M., Gulesci, S., Rasul, I. and Sulaiman, M., 2012. Empowering adolescent girls: Evidence from a randomized control trial in Uganda. Washington, DC: World Bank.

Baryamutuma, R., Nabaggala, R., Muhairwe, L. B. and Baingana, F. 2010. Factors influencing sexual behaviours among adolescents living with HIV and AIDS in Uganda. 18th International AIDS Conference - AIDS2010, Vienna.

Bermudez, L. G., Ssewamala, F. M., Neilands, T. B., Lu, L., Jennings, L., Nakigozi, G., Mellins, C. A., Mckay, M. and Mukasa, M. (2018). Does economic strengthening improve viral suppression among adolescents living with HIV? Results from a cluster randomized trial in Uganda. AIDS and Behavior, 22(11), pp. 3763-3772.

Bernays, S., Paparini, S., Gibb, D. and Seeley, J., 2016. When information does not suffice: Young people living with HIV and communication about ART adherence in the clinic. Vulnerable Children and Youth Studies, pp. 1-9, February.

Bhana, A., Mellins, C.A., Petersen, I., Alicea, S., Myeza, N., Holst, H., Abrams, E., John, S., Chhagan, M., Nestadt, D. F. and Leu, C. S., 2014. The VUKA family program: Piloting a family-based psychosocial intervention to promote health and mental health among HIV infected early adolescents in South Africa. AIDS Care, 26(1), pp. 1-11.

Busza, J., Besana, G.V., Mapunda, P. and Oliveras, E., 2014. Meeting the needs of adolescents living with HIV through home based care: Lessons learned from Tanzania. Children and Youth Services Review, 45, pp. 137-142, February. 
Campbell, C., Skovdal, M., Mupambireyi, Z., Madanhire, C., Nyamukapa, C. and Gregson, S., 2012. Building adherence-competent communities: Factors promoting children's adherence to anti-retroviral HIV/AIDS treatment in rural Zimbabwe. Health and Place, 18(2), pp. 123-131.

Cho, H., Hallfors, D. D., Mbai, I. I., Itindi, J., Milimo, B. W., Halpern, C. T. and Iritani, B.J., 2011. Keeping adolescent orphans in school to prevent human immunodeficiency virus infection: Evidence from a randomized controlled trial in Kenya. Journal of Adolescent Health, 48(5), pp. 523-526.

Cluver, L. D., Hodes, R.J., Toska, E., Kidia, K. K., Orkin, F. M., Sherr, L. and Meinck, F., 2015. "HIV is like a tsotsi. ARVs are your guns": Associations between HIVdisclosure and adherence to antiretroviral treatment among adolescents in South Africa. AIDS, 29, pp. S57-S65, April.

Cluver, L. D., Meinck, F., Toska, E., Orkin, F. M., Hodes, R. and Sherr, L., 2018. Multitype violence exposures and adolescent antiretroviral nonadherence in South Africa. AIDS, 32(8), pp. 975-983.

Cluver, L.D., Orkin, M.F., Yakubovich, A.R. and Sherr, L., 2016a. Combination social protection for reducing HIV-risk behavior amongst adolescents in South Africa. Journal of Acquired Immune Deficiency Syndromes, 72(1), p. 96.

Cluver, L. D., Toska, E., Orkin, F. M., Meinck, F., Hodes, R., Yakubovich, A. R. and Sherr, L., 2016b. Achieving equity in HIV-treatment outcomes: Can social protection improve adolescent ART-adherence in South Africa? AIDS Care, 28(Suppl 2), pp. 73-82.

Coates, T.J., Richter, L. and Caceres, C., 2008. Behavioural strategies to reduce HIV transmission: How to make them work better. Lancet, 372(9639), pp. 669-684.

Cohen, M.S., Smith, M. K., Muessig, K. E., Hallett, T. B., Powers, K. A. and Kashuba, A.D., 2013. Antiretroviral treatment of HIV-1 prevents transmission of HIV-1: Where do we go from here? Lancet, 382(9903). pp. 1515-1524.

Delany-Moretlwe, S., Cowan, F.M., Busza, J., Bolton-Moore, C., Kelley, K. and Fairlie, L., 2015. Providing comprehensive health services for young key populations: Needs, barriers and gaps. JIAS: Journal of the International AIDS Society, 18(2 Suppl 1), p. 19833.

Denison, J.A., Banda, H., Dennis, A.C., Packer, C., Nyambe, N., Stalter, R.M., Mwansa, J.K., Katayamoyo, P. and McCarraher, D. R., 2015. "The sky is the limit": Adhering to antiretroviral therapy and HIV self-management from the perspectives of adolescents living with HIV and their adult caregivers. JIAS: Journal of the International AIDS Society, 18(1), p. 19358.

Devereux, S. and Sabates-Wheeler, R., 2004. Transformative social protection. IDS working paper.

Duflo, E., Dupas, P. and Kremer, M., 2015. Education, HIV, and early fertility: Experimental evidence from Kenya. American Economic Review, 105(9), pp. 2757-2797.

Emenyonu, N., Muyindike, W., Habyarimana, J., Pops-Eleches, C., Thirumurthy, H., Ragland, K. and Bangsberg, D. R., 2010. Cash transfers to cover clinic transportation costs improve adherence and retention in care in a HIV treatment program in rural Uganda. 17th Conference on Retroviruses and Opportunistic Infections, San Francisco, CA. Garcia, M. and Moore, C. M., 2012. The cash dividend: The rise of cash transfer programs in sub-Saharan Africa. Washington, DC: World Bank.

Gillespie, S., Kadiyala, S. and Greener, R., 2007. Is poverty or wealth driving HIV transmission? AIDS, 21 (Suppl 7), pp. S5-S16.

Gittings, L., Toska, C., Hodes, R., Cluver, L., Zungu, N., Govender, K., Chademana, K.E. and Gutiérrez, V.E., 2016. Resourcing resilience: The case for social protection 
for adherence and HIV-related outcomes in children and adolescents in Eastern and Southern Africa. RIATT-ESA Report, June. Available at: https://ovcsupport.org/ resource/resourcing-resilience-the-case-for-social-protection-for-adherence-andhiv-related-outcomes-in-children-and-adolescents-in-eastern-and-southern/.

Govender, K., Masebo, W. G., Nyamaruze, P., Cowden, R. G., Schunter, B. T. and Bains, A., 2018. HIV prevention in adolescents and young people in the Eastern and Southern African region: A review of key challenges impeding actions for an effective response. The Open AIDS Journal, 12, p. 53.

Govender, K., Reardon, C., Quinlan, T. and George, G., 2014. Children's psychosocial wellbeing in the context of HIV/AIDS and poverty: A comparative investigation of orphaned and non-orphaned children living in South Africa. BMC Public Health, 14(1), p. 615.

Grimwood, A., Fatti, G., Mothibi, E., Malahlela, M., Shea, J. and Eley, B., 2012. Community adherence support improves programme retention in children on antiretroviral treatment: A multicentre cohort study in South Africa. JIAS: Journal of the International AIDS Society, 15(2), pp. 1-9.

Handa, T., Halpern, C. T., Pettifor, A. and Thirumurthy, H., 2014. The government of Kenya's cash transfer program reduces the risk of sexual debut among young people age 15-25. PLoS One, 9(1), p.e85473.

Hodes, R., 2013. "You know what a bad person you are?" HIV, abortion, and reproductive health care for women in South Africa. In R. A. Smith, ed. Global HIV/ AIDS politics, policy and activism: Persistent challenges and emerging issues. Santa Barbara, CA: Praeger, pp. 233-252, 239-241.

Hodes, R., Doubt, J., Toska, E., Vale, B., Zungu, N. and Cluver, L., 2018b. The stuff that dreams are made of: HIV-positive adolescents' aspirations for development. JIAS: Journal of the International AIDS Society, 21 (Suppl 1), p. e25057.

Hodes, R. and Naimak, T. 2011. Piloting antiretroviral therapy in South Africa: The role of partnerships in the Western Cape's provincial roll-out. African Journal of AIDS Research, 10(4), pp. 415-425.

Hodes, R., Vale, B., Toska, E., Cluver, L., Dowse, R. and Ashorn, M., 2018a. Yummy or crummy? The multisensory components of medicines-taking among HIVpositive youth. Global Public Health, 14(2), pp. 284-299.

Honorati, M., Gentilini, U. and Yemtsov, R. G., 2015. The state of social safety nets 2015. Washington, DC: International Bank for Reconstruction and Development / The World Bank. Available at http://documents.worldbank.org/curated/ en/2015/07/24741765/state-social-safety-nets-2015

Hudelson, C. and Cluver, L. D., 2015. Factors associated with adherence to antiretroviral therapy among adolescents living with HIV/AIDS in low- and middle-income countries: A systematic review. AIDS Care, 27(7), pp. 805-816.

Iwuji, C. C., Orne-Gliemann, J., Larmarange, J., Balestre, E., Thiebaut, R., Tanser, F., Okesola, N., Makowa, T., Dreyer, J., Herbst, K. and McGrath, N., 2018. Universal test and treat and the HIV epidemic in rural South Africa: A phase 4, open-label, community cluster randomised trial. Lancet HIV, 5(3), pp. e116-e125.

Karim, Q.A., 2015. Impact of conditional cash incentives on HSV-2 and HIV in rural high school students in South Africa: The CAPRISA 007 cluster randomized controlled trial. 8th IAS Conference on HIV Pathogenesis, Treatment \& Prevention, Vancouver, Canada.

Kidman, R., Nachman, S., Dietrich, J., Liberty, A. and Violari, A., 2018. Childhood adversity increases the risk of onward transmission from perinatal HIV-infected adolescents and youth in South Africa. Child Abuse and Neglect, 79, pp. 98-106. 
Lightfoot, M.A., Rotheram-Borus, M.J. and Tevendale, H., 2007. An HIV-preventive intervention for youth living with HIV. Behavior Modification, 31, pp. 345-363.

Marhefka, S.L., Elkington, K., Dolezal, C. and Mellins, C., 2010. Transmission risk behaviour among youth living with perinatally acquired HIV: Are nonadherent youth more likely to engage in sexual behavior? Society for Adolescent Medicine Annual Meeting Program Issue: Adolescent Clinical Care: Integrating Art E Science, p. S29.

Mavhu, W., Berwick, J., Chirawu, P., Makamba, M., Copas, A., Dirawo, J., Willis, N., Araya, R., Abas, M.A., Corbett, E. L. and Mungofa, S., 2013. Enhancing psychosocial support for HIV positive adolescents in Harare, Zimbabwe. PLoS One, 8(7), p. e70254.

Mbalinda, S.N., Kiwanuka, N., Kaye, D. K. and Eriksson, L.E., 2015. Reproductive health and lifestyle factors associated with health-related quality of life among perinatally HIV-infected adolescents in Uganda. Health and Quality of Life Outcomes, 13(1), p. 170.

Mupambireyi, Z., Bernays, S., Bwakura-Dangarembizi, M. and Cowan, F. M., 2014. "I don't feel shy because I will be among others who are just like me ...": The role of support groups for children perinatally infected with HIV in Zimbabwe. Children and Youth Services Review, 45, pp. 106-113.

Nachega, J. B., Hislop, M., Nguyen, H., Dowdy, D. W., Chaisson, R.E., Regensberg, L., Cotton, M. and Maartens, G., 2009. Antiretroviral therapy adherence, virologic and immunologic outcomes in adolescents compared with adults in Southern Africa. Journal of Acquired Immune Deficiency Syndromes, 51(1), pp. 65-71.

National Planning Commission, Department of the Presidency, Republic of South Africa, 2012. National development plan 2030: Our future, make it work. Available at www.gov.za/sites/default/files/gcis_document/201409/ndp-2030-our-futuremake-it-workr.pdf, accessed 4 April 2019.

Nattrass, N. and Gonsalves, G., 2009. Economics and the backlash against AIDS-specific funding. Cape Town: University of Cape Town.

Nhamo, D., 2013. Shaping the health of adolescents in Zimbabwe (SHAZ!) Key findings and recommendations from an economic and lifeskills intervention addressing SRH and HIV issues among female adolescents. International AIDS Conference. Available at: https:// www.avac.org/sites/default/files/event_files/SHAZ\%21\%20webinar\%20slides.pdf.

Obare, F., Birungi, H. and Kavuma, L., 2011. Barriers to sexual and reproductive health programming for adolescents living with HIV in Uganda. Population Research and Policy Review, 30, pp. 151-163.

O'Hare, B.A., Venables, J., Nalubeg, J.F., Nakakeeto, M., Kibirige, M. and Southall, D. P., 2005. Home-based care for orphaned children infected with HIV/AIDS in Uganda. AIDS Care, 17(4), pp. 443-450.

Orne-Gliemann, J., Larmarange, J., Boyer, S., Iwuji, C., McGrath, N., Bärnighausen, T., Zuma, T., Dray-Spira, R., Spire, B., Rochat, T. and Lert, F., 2015. Addressing social issues in a universal HIV test and treat intervention trial (ANRS 12249 TasP) in South Africa: Methods for appraisal. BMC Public Health, 15(1), p. 209.

Padian, N.S., McLoy, S.I., Balkus, J.E. and Wasserheit, J.N., 2010. Weighing the gold in the gold standard: Challenges in HIV prevention research. AIDS, 24(5), pp. 621-635.

Paediatric AIDS Treatment for Africa \& World Health Organization, 2015. Adolescent Africa: A situational analysis of adolescent HIV-treatment and care in sub-Saharan Africa. Cape Town: World Health Organization.

Parker, L., Maman, S., Pettifor, A., Chalachala, J.L., Edmonds, A., Golin, C.E., Moracco, K. and Behets, F., 2013. Adaptation of a U.S. evidence-based positive 
prevention intervention for youth living with HIV/AIDS in Kinshasa, democratic republic of the Congo. Evaluation and Program Planning, 36, pp. 124-135.

Paterson, D. L., Swindells, S., Mohr, J., Brester, M., Vergis, E. N., Squier, C., Wagener, M. M. and Singh, N., 2000. Adherence to protease inhibitor therapy and outcomes in patients with HIV infection. Annals of Internal Medicine, 133, pp. 21-30.

PEPFAR, 2015. Preventing HIV in adolescent girls and young women: Guidance for PEPFAR country teams on the DREAMS partnership. Washington, DC: PEPFAR.

Pettifor, A. E., Levandowski, B. A., MacPhail, C., Padian, N. S., Cohen, M. S. and Rees, H.V., 2008. Keep them in school: The importance of education as a protective factor against HIV infection among young South African women. International Journal of Epidemiology, 37(6), pp. 1266-1273.

Pettifor, A. E., Nguyen, N. L., Celum, C., Cowan, F. M., Go, V. and Hightow-Weidman, L., 2015. Tailored combination prevention packages and PrEP for young key populations. Journal of the International AIDS Society, 18(2), pp. 8-22.

Phillips, H. 2014. The return of the Pholela experiment: Medical history and primary health care in post-apartheid South Africa. American Journal of Public Health, 104(10), pp. 1872-1876.

Remme, M., Vassall, A., Lutz, B., Luna, J. and Watts, C., 2014. Financing structural interventions: Going beyond HIV-only value for money assessments. AIDS, 28(3), pp. $425-434$.

Ridgeway, K., Dulli, L.S., Murray, K. R., Silverstein, H., Dal Santo, L., Olsen, P., de Mora, D.D. and McCarraher, D.R., 2018. Interventions to improve antiretroviral therapy adherence among adolescents in low- and middle-income countries: A systematic review of the literature. PLoS One, 13(1), p.e0189770.

Schneider, H., Hlophe, H. and van Rensburg, D. 2008. Community health workers and the response to HIV/AIDS in South Africa: Tensions and prospects. Health Policy and Planning, 23(3), pp. 179-187.

Senyonyi, R.M., Underwood, L.A., Suarez, E., Musisi, S. and Grande, T.L., 2012. Cognitive behavioral therapy group intervention for HIV transmission risk behavior in perinatally infected adolescents. Health, 4(12), pp. 1334-1345.

Shisana, O. et al., 2014. South African national HIV prevalence, incidence and behaviour survey, 2012. Cape Town: HSRC Press.

Snyder, K., Wallace, M., Duby, Z., Aquino, L. D., Stafford, S., Hosek, S., Futterman, D. and Bekker, L. G., 2014. Preliminary results from Hlanganani (coming together): A structured support group for HIV-infected adolescents piloted in Cape Town, South Africa. Children and Youth Services Review, 45, pp. 114-121.

Ssewamala, F. 2013. Evaluating a youth-focused economic empowerment approach to HIV treatment adherence - study protocol. Available at: https://clinicaltrials.gov/ct2/ show/NCT01790373, accessed November 30, 2018.

Standish-White, J., 2018. A cost-effectiveness analysis of stack: Maximising retention in care for adolescents living with HIV in South Africa. Oxford: University of Oxford Press.

Strasser, S. and Gibbons, S., 2014. The development of HIV-related mental health and psychosocial services for children and adolescents in Zambia: The case for learning by doing. Children and Youth Services Review, 45, pp. 150-157.

Toska, E. and Cluver, L. D., 2018. Barriers to U=U for adolescents living with HIV: Predictors of high HIV-transmission risk from a longitudinal cohort study. 2nd International Workshop on HIV Adolescence - Challenges \& Solutions, Reviews in Antiviral Therapy \& Infectious Diseases, Cape Town, South Africa, p. 31.

Toska, E., Cluver, L. D., Boyes, M. E., Isaacsohn, M., Hodes, R. and Sherr, L., 2017 a. School, supervision and adolescent-sensitive clinic care: Combination social 
protection and reduced unprotected sex among HIV-positive adolescents in South Africa. AIDS and Behavior, 21 (9), 2746-2759.

Toska, E., Cluver, L.D., Hodes, R. and Kidia, K. K., 2015. Sex and secrecy: How HIV-status disclosure affects safe sex among HIV-positive adolescents. AIDS Care, 27 (Supp 1), pp. 47-58.

Toska, E., Pantelic, M., Meinck, F., Keck, K., Haghighat, R. and Cluver, L., 2017b. Sex in the shadow of HIV: A systematic review of prevalence, risk factors, and interventions to reduce sexual risk-taking among HIV-positive adolescents and youth in sub-Saharan Africa. PLoS One, 12(6), p. e0178106. doi:10.1371/journal. pone.0178106.

UNAIDS, 2014. HIV and social protection guidance note. Geneva, Switzerland: UNAIDS.

UNAIDS, 2017. UNAIDS data 2017. Geneva, Switzerland: UNAIDS.

UNAIDS, 2018. Miles to go: Closing gaps, breaking barriers, righting injustices. Geneva, Switzerland: UNAIDS.

UNICEF, 2015. Social protection evaluation synthesis UNICEF evaluation management response. New York: UNICEF.

UNICEF, 2017. Children and AIDS: Statistical update. New York: UNICEF.

Vale, B., Hodes, R. and Cluver, L., 2017. Negotiations of blame and care among HIVpositive mothers and daughters in South Africa's Eastern Cape. Medical Anthropology Quarterly, 31(4), pp. 519-536.

Van Winghem, J., Telfer, B., Reid, T., Ouko, J., Mutunga, A., Jama, Z. and Vakil, S., 2008. Implementation of a comprehensive program including psycho-social and treatment literacy activities to improve adherence to HIV care and treatment for a pediatric population in Kenya. BMC Pediatrics, 8, p. 52.

Visser, M., Zungu, N. and Ndala-Magoro, N., 2015. ISIBINDI, creating circles of care for orphans and vulnerable children in South Africa: Post-programme outcomes. AIDS Care: Psychological and Socio-Medical Aspects of AIDS/HIV, 27(8), pp. 1014-1019.

WHO, 2015. Guidelines on when to start antiretroviral therapy and on a pre-exposure prophylaxis for HIV. Geneva, Switzerland: World Health Organization.

World Bank, 2015. The state of social safety nets 2015. Washington, DC: World Bank. 